\title{
LA-UR-16-23092
}

Approved for public release; distribution is unlimited.

Title: $\quad$ Beam line shielding calculations for an Electron Accelerator Mo-99 production facility

Author(s): $\quad$ Mocko, Michal

Intended for: Report

Issued:

2016-05-03 
Disclaimer:

Los Alamos National Laboratory, an affirmative action/equal opportunity employer, is operated by the Los Alamos National Security, LLC for the National Nuclear Security Administration of the U.S. Department of Energy under contract DE-AC52-06NA25396. By approving this article, the publisher recognizes that the U.S. Government retains nonexclusive, royalty-free license to publish or reproduce the published form of this contribution, or to allow others to do so, for U.S. Government purposes. Los Alamos National Laboratory requests that the publisher identify this article as work performed under the auspices of the U.S. Department of Energy. Los Alamos National Laboratory strongly supports academic freedom and a researcher's right to publish; as an institution, however, the Laboratory does not endorse the viewpoint of a publication or guarantee its technical correctness. 


\section{Beam line shielding calculations for an Electron Accelerator Mo-99 production facility}

M. Mocko

April 2016

\section{Introduction}

The purpose of this study is to evaluate the photon and neutron fields in and around the latest beam line design [1] for the Mo-99 production facility. The radiation dose to the beam line components (quadrupoles, dipoles, beam stops and the linear accelerator) are calculated in the present report. The beam line design assumes placement of two cameras: infra red (IR) and optical transition radiation (OTR) for continuous monitoring of the beam spot on target during irradiation. The cameras will be placed off the beam axis offset in vertical direction. We explored typical shielding arrangements for the cameras and report the resulting neutron and photon dose fields.

\section{Layout}

We implemented simplified beam line geometry into MCNPX based on the previous report [1]. In Fig. 1 we compare the beam line geometry from Ref. [1] to our implementation in MCNPX (lower panel). In the MCNPX implementation we labeled the principal components: quadrupoles, dipoles, beam stops, and linear accelerator (linac) modules. All of the above mentioned components are implemented with equivalent size and homogenized material composition of typical realistic beam line components. Since there is no detailed description of the beam line components we did not attempt to model a specific design, rather provide bounding estimates of the radiation fields in a particular layout (based on Ref. [1]). The geometry layout contains a total of 8 quadrupoles, 3 dipoles, two beam stops and 5 modules of the linear accelerator. All presented calculations assumed two electron beams of 120 $\mathrm{kW}$ each at $42 \mathrm{MeV}$ impinging on a solid Mo-100 target. 

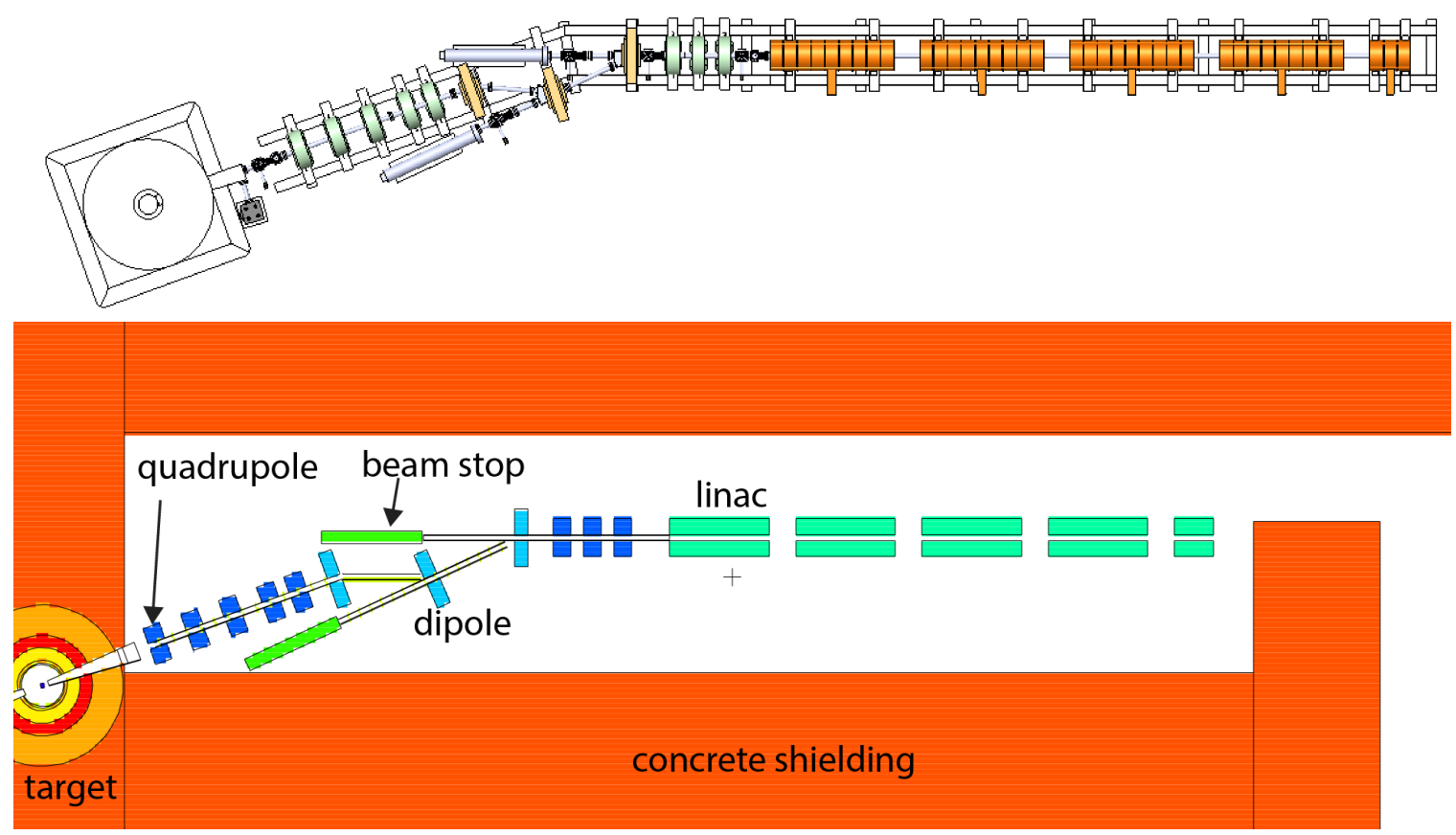

Figure 1: Beam line geometry from the design report [1] shown above is compared to the MCNPX geometry implementation displayed below.

For the IR camera placement we assumed a placement directly below the beam axis right inside of the beam line cave (see Fig. 2: label IR). For the OTR camera we explored two potential placements. First one approximately $35 \mathrm{~cm}$ off the beam axis in horizontal plane next to the IR camera (see Fig. 2: label OTR1). The second position is further down stream, just below the first dipole from the target on beam axis (see Fig. 2: label OTR2). All camera locations are assumed to be $131 \mathrm{~cm}$ below the beam axis. Two of them are directly below the beam axis (IR and OTR2) and one is below a location off the beam axis (OTR1).

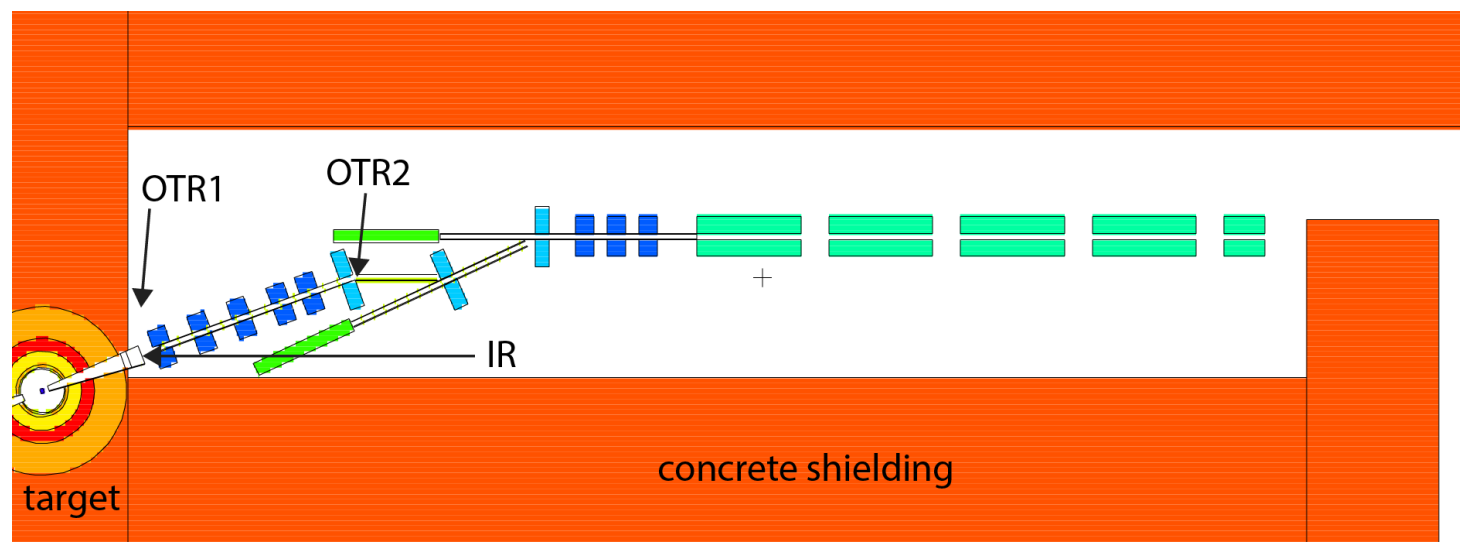

Figure 2: Positioning of the camera locations. The cameras are assumed to be placed on the flow approximately $131 \mathrm{~cm}$ below the beam axis at locations indicated by labels (see text for details). 


\section{Results and Discussion}

In Fig. 3 we show the dose rate equivalent fields for neutrons and in Fig. 4 we show the photon dose rate equivalent fields.

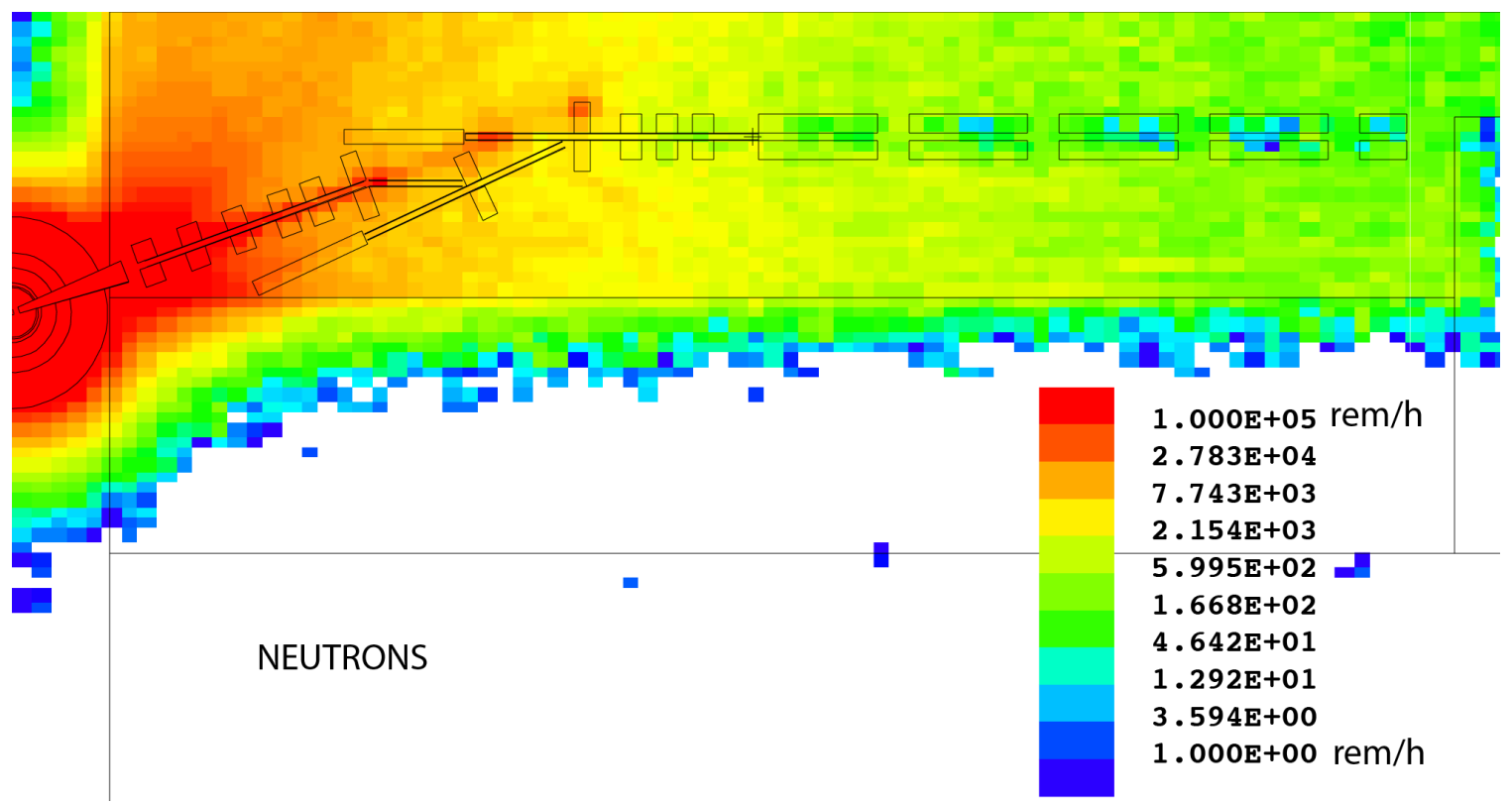

Figure 3: Neutron dose rate equivalents around the beam line components.

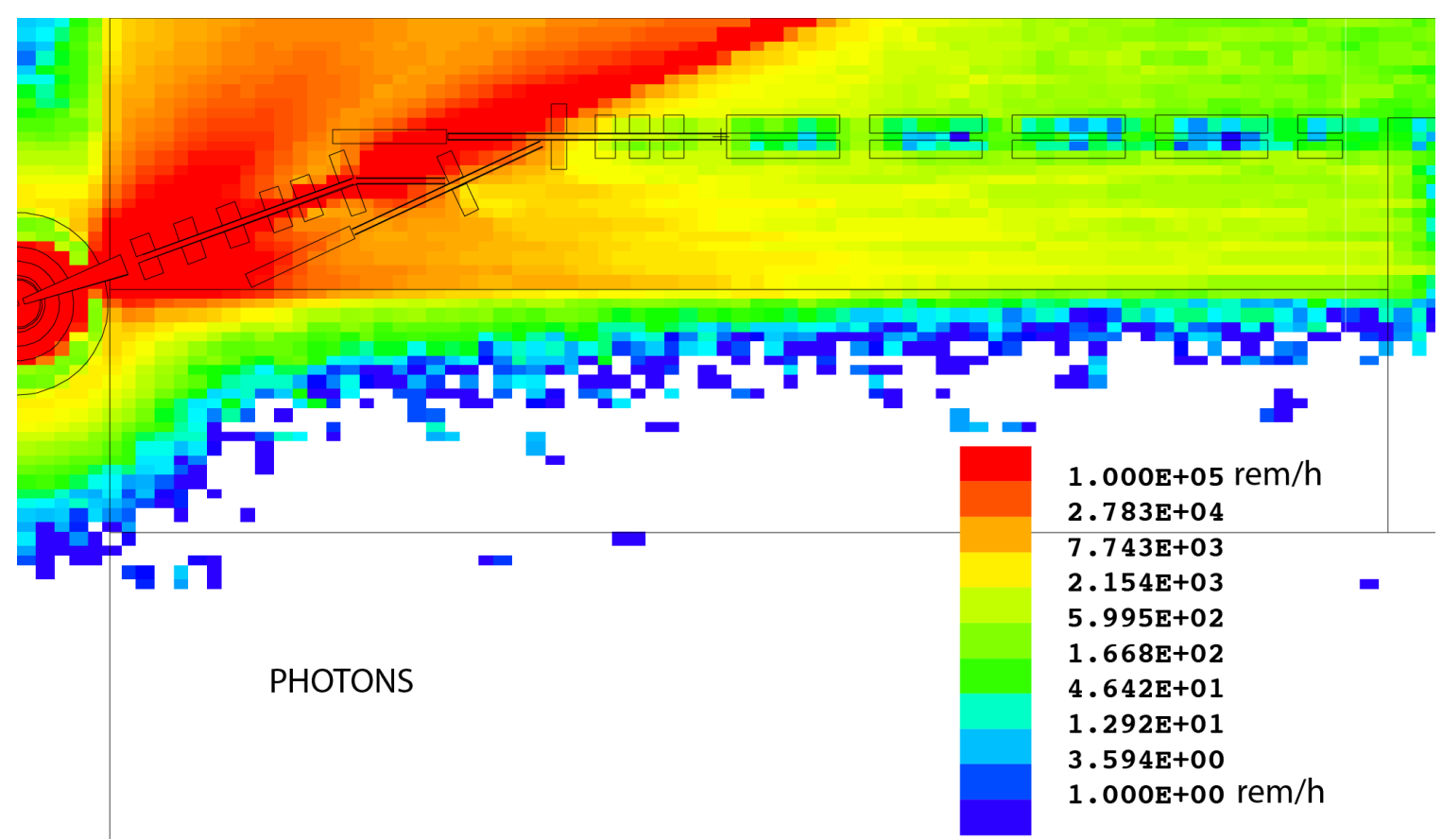

Figure 4: Photon dose rate equivalents around the beam line components. 
There is a strong directional dependence for the photon fields whereas the neutrons are distributed a little more isotropically (as was described in the previous shielding report [2]). We notice that the first quadrupole series will be in much higher dose fields as compared to the rest of the beam line components.

In the first set of calculations we calculated the radiation dose from neutrons and photons to the beam line components. In Fig. 5 we show the unique labels assigned to each quadrupole, dipole, beam stop and linac module.

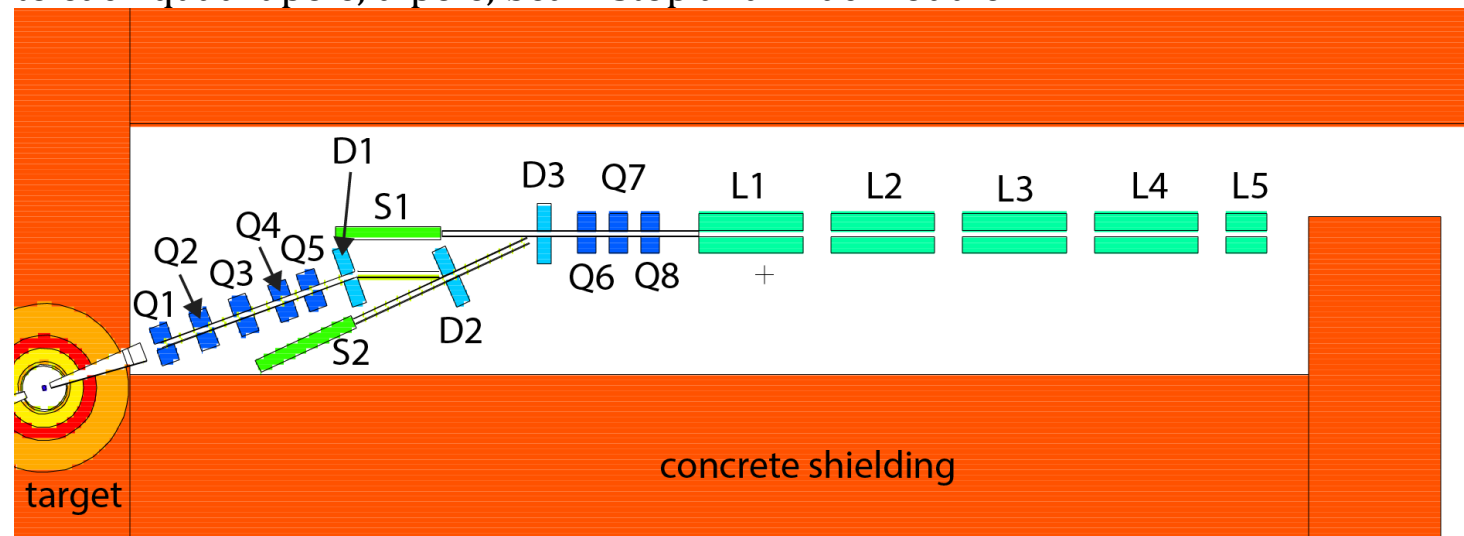

Figure 5: Unique labels assigned to each of the principal beam line components studied in the present report.

In Table 1 we list the resulting radiation dose in units of $\mathrm{rad} / \mathrm{h}$ for individual beam line components as labeled in Fig. 5.

Table 1: Radiation dose for individual beam line components induced by neutrons and protons. All data are listed in units of rad/h assuming two electron beams of $120 \mathrm{~kW}$ each at $42 \mathrm{MeV}$.

\begin{tabular}{|l|l|l|l|l|}
\hline \multirow{2}{*}{ Label } & \multicolumn{3}{l}{ No shutter } & \multicolumn{2}{l|}{ Tungsten shutter } \\
\cline { 2 - 5 } & neutrons & photons & neutrons & photons \\
\hline Q1 & $1.40 \mathrm{E}+03$ & $2.02 \mathrm{E}+07$ & $2.89 \mathrm{E}+02$ & $5.56 \mathrm{E}+05$ \\
\hline Q2 & $2.12 \mathrm{E}+02$ & $1.98 \mathrm{E}+06$ & $3.23 \mathrm{E}+01$ & $4.86 \mathrm{E}+04$ \\
\hline Q3 & $4.10 \mathrm{E}+01$ & $2.05 \mathrm{E}+05$ & $8.52 \mathrm{E}+00$ & $4.29 \mathrm{E}+03$ \\
\hline Q4 & $1.65 \mathrm{E}+01$ & $3.73 \mathrm{E}+04$ & $4.91 \mathrm{E}+00$ & $1.21 \mathrm{E}+03$ \\
\hline Q5 & $1.15 \mathrm{E}+01$ & $1.61 \mathrm{E}+04$ & $3.42 \mathrm{E}+00$ & $7.55 \mathrm{E}+02$ \\
\hline Q6 & $1.16 \mathrm{E}+01$ & $1.50 \mathrm{E}+03$ & $6.12 \mathrm{E}-01$ & $2.02 \mathrm{E}+02$ \\
\hline Q7 & $8.19 \mathrm{E}-01$ & $4.88 \mathrm{E}+02$ & $5.23 \mathrm{E}-01$ & $1.76 \mathrm{E}+02$ \\
\hline Q8 & $7.78 \mathrm{E}-01$ & $4.04 \mathrm{E}+02$ & $4.54 \mathrm{E}-01$ & $1.62 \mathrm{E}+02$ \\
\hline D1 & $1.18 \mathrm{E}+01$ & $1.29 \mathrm{E}+04$ & $3.92 \mathrm{E}+00$ & $7.15 \mathrm{E}+02$ \\
\hline D2 & $6.83 \mathrm{E}+00$ & $4.98 \mathrm{E}+04$ & $2.78 \mathrm{E}+00$ & $6.16 \mathrm{E}+02$ \\
\hline D3 & $6.02 \mathrm{E}+00$ & $8.22 \mathrm{E}+04$ & $1.31 \mathrm{E}+00$ & $6.59 \mathrm{E}+02$ \\
\hline S1 & $2.39 \mathrm{E}+01$ & $1.40 \mathrm{E}+05$ & $1.08 \mathrm{E}+01$ & $6.46 \mathrm{E}+02$ \\
\hline S2 & $5.86 \mathrm{E}+01$ & $2.39 \mathrm{E}+04$ & $3.52 \mathrm{E}+01$ & $3.60 \mathrm{E}+02$ \\
\hline L1 & $4.65 \mathrm{E}-01$ & $2.25 \mathrm{E}+02$ & $3.09 \mathrm{E}-01$ & $1.04 \mathrm{E}+02$ \\
\hline L2 & $2.28 \mathrm{E}-01$ & $1.47 \mathrm{E}+02$ & $1.89 \mathrm{E}-01$ & $6.75 \mathrm{E}+01$ \\
\hline L3 & $1.51 \mathrm{E}-01$ & $9.16 \mathrm{E}+01$ & $1.06 \mathrm{E}-01$ & $4.84 \mathrm{E}+01$ \\
\hline L4 & $9.06 \mathrm{E}-02$ & $6.24 \mathrm{E}+02$ & $8.21 \mathrm{E}-02$ & $3.54 \mathrm{E}+01$ \\
\hline L5 & $8.92 \mathrm{E}-02$ & $6.25 \mathrm{E}+02$ & $1.12 \mathrm{E}-01$ & $3.79 \mathrm{E}+01$ \\
\hline
\end{tabular}


The radiation dose calculations were carried out for two scenarios: unmitigated, where there is a direct line of sight between the first section of the beam line (Q1Q5) and the target and another one with a $5-\mathrm{cm}$ thick tungsten piece shielding the beam line components from the target. This tungsten layer represents a shutter that could be implemented as a rotating tungsten wheel with alternating openings to let the electron beam through. This report will not elaborated on potential implementation details, rather than provide quantitative assessment of potential benefits of such a system.

We note that dose to the beam line components is vastly dominated by photons, we see a difference of more than 4 orders of magnitude for the unmitigated scenario for the first dipole. The radiation doses fall fairly quickly as a function of distance from the production target. Regardless, the radiation dose to the first set of quadrupoles is substantial ( $1 \mathrm{E}+04-2 \mathrm{E}+07 \mathrm{rad} / \mathrm{h}$ for the no shutter case). By introducing the tungsten shutter we are able to lower the radiation dose to the first set of quadrupoles by almost two orders of magnitude (down to $8 \mathrm{E}+02-6 \mathrm{E}+05 \mathrm{rad} / \mathrm{h}$ ).

In the second step we focused on assessing the radiation dose fields for the potential placements of two beam-profile monitoring cameras. As mentioned above we explored one position for the IR camera (IR in Fig. 2) and two potential locations for the OTR camera (OTR1 and OTR2 in Fig. 2). Radiation hardness for these cameras is assumed to be approximately $1 \mathrm{rad} / \mathrm{h}$ in photon fields [3]. The neutron radiation hardness is not properly assessed at this time. From the results listed in Table 1 we note that the ratio between photon and neutron radiation fields off the direct line of sight axis is approximately a factor of 100 (two orders of magnitude).

Table 2: Radiation dose fields at the camera locations labeled as in Figure 2. All data in the units of rad/h assuming full beam power of two electron beams at $120 \mathrm{~kW}$ at $42 \mathrm{MeV}$.

\begin{tabular}{|l|l|l|l|l|}
\hline \multirow{2}{*}{ Location } & \multicolumn{2}{|l|}{ No shutter } & \multicolumn{2}{l|}{ Tungsten shutter } \\
\cline { 2 - 5 } & Neutrons & Photons & Neutrons & Photons \\
\hline IR & 22.8 & 4230 & 9.2 & 678 \\
\hline OTR1 & 21.4 & 3790 & 10.7 & 710 \\
\hline OTR2 & 17.6 & 7290 & 15.6 & 2400 \\
\hline
\end{tabular}

Table 2 summarizes our results for the three potential camera locations without any additional shielding surrounding the camera. We see that the ratio between the photon and neutron dose fields is more than a factor of 100 at all investigated locations. We also conclude that the addition of the tungsten shutter (as described above) results in significant lowering of the photon dose. It is clear that additional local shielding is necessary for all camera locations if one wants to approach the desirable $1 \mathrm{rad} / \mathrm{h}$ photon fields. At the same time we caution that the amount of local camera shielding will be substantial as we need to reduce the photon fields by three orders of magnitude!

In the next step we investigate a variety of shielding materials and thicknesses assuming $3 \mathrm{~cm}$ diameter opening in the vertical direction for the camera to retain 
the necessary line of sight. The photon dose fields at OTR2 location tend to be much higher than OTR1 location due to larger scatter of the photons produced in the target on the quadrupoles and other beam line components. A typical arrangement of the cameras and shielding is shown in Figure 6. Please note that IR and OTR1 locations are in the same shielding enclosure as they are too close to facilitate independent shielding.

Table 3: Radiation dose fields at the camera locations labeled as in Figure 2. All data are in the units of $\mathrm{rad} / \mathrm{h}$.

\begin{tabular}{|l|l|l|l|l|}
\hline \multirow{2}{*}{ Location } & \multicolumn{2}{|l|}{$\begin{array}{l}15 \mathrm{~cm}+15 \mathrm{~cm} \text { lead and borated } \\
\text { poly, with } 3 \mathrm{~cm} \text { opening }\end{array}$} & \multicolumn{2}{l|}{$\begin{array}{l}15 \mathrm{~cm}+15 \mathrm{~cm} \text { lead and borated } \\
\text { poly, no opening }\end{array}$} \\
\cline { 2 - 5 } & Neutrons & Photons & Neutrons & Photons \\
\hline IR & 1.6 & 12 & 1.6 & 12 \\
\hline OTR1 & 0.8 & 10 & 0.8 & 0.6 \\
\hline OTR2 & 0.8 & 156 & 0.6 & 1.6 \\
\hline
\end{tabular}

The results listed in Table 3 for the case of no vertical line-of-sight opening for the cameras is not realistic, but it shows the maximum efficacy of the shielding in the current configuration. We conclude that the IR camera location photon dose and neutron dose are dominated by the radiation streaming through the local target

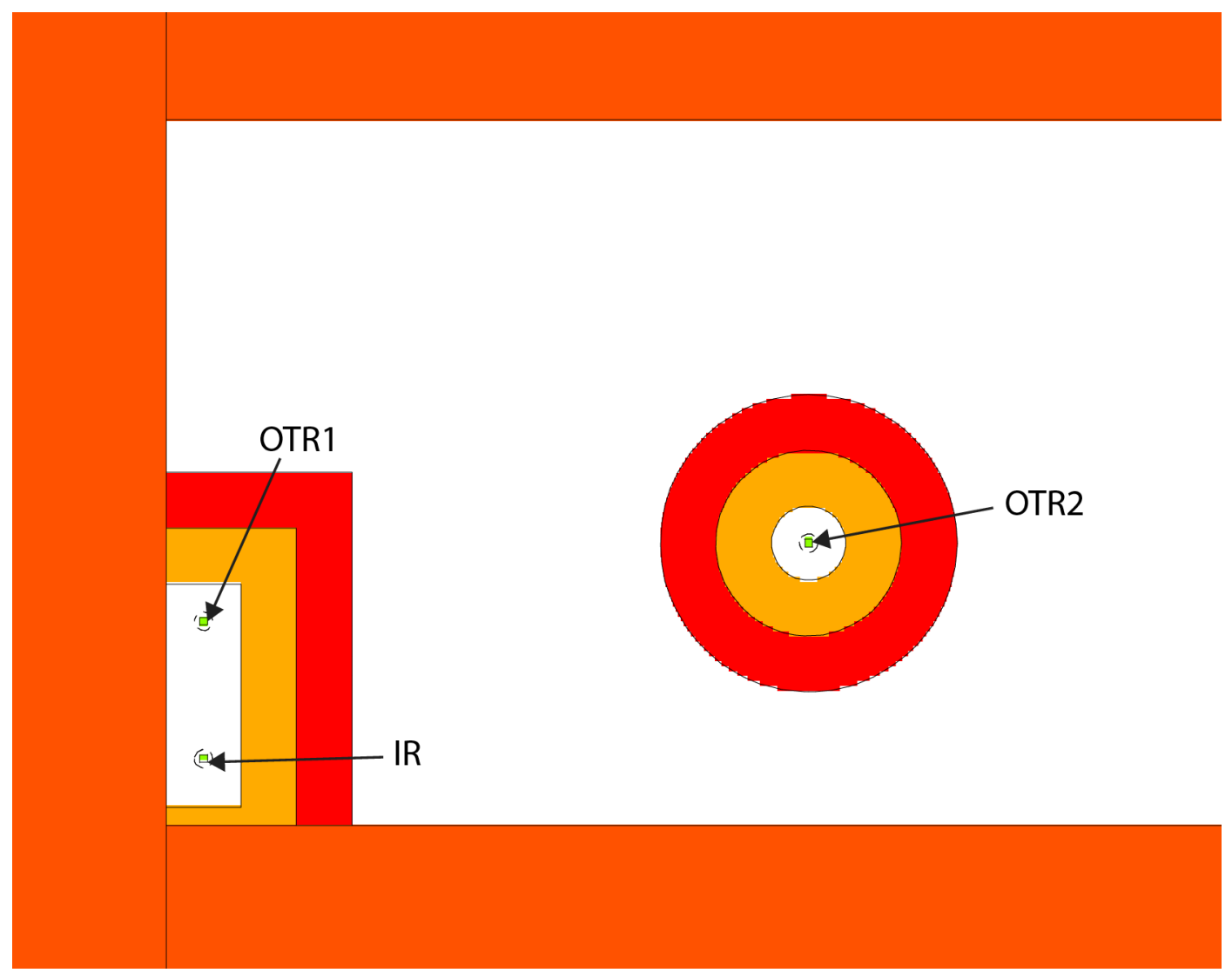

Figure 6: Layout of the three camera locations shown in MCNPX geometry at the camera level $(131 \mathrm{~cm}$ below the beam axis). 
shielding and the vertical line-of-sight camera penetration does not cause a significant effect. Whereas for 0TR1 location we notice a rather significant difference for photon dose between opening and not opening case (10 versus 0.6 $\mathrm{rad} / \mathrm{h}$, see Table 3 ). We note similarly, a large difference for OTR2 camera location between the case with and without vertical opening. Next, we ran a model with steel pipes shielding the direct line of sight for the cameras to assess how much they reduce the streaming radiation. Addition of these shielding lines does not help for IR, OTR1 positions (reducing the dose rates by 10$30 \%$ ). We noticed a significant reduction of the photon dose at OTR2 position: from $156 \mathrm{rad} / \mathrm{h}$ (bare opening) down to $39 \mathrm{rad} / \mathrm{h}$ (with added shielding pipe).

\section{Conclusions}

We have carried out an initial shielding assessment for the beam line components based on the latest conceptual design [1]. We have calculated neutron and photon dose to the beam line components (quadrupoles, dipoles, beam dumps and linear accelerator modules). We conclude our study with the following points:

- Photon dose rates to the first set of quadrupoles are substantial. They are severe for the first quadrupole in particular. Some sort of additional shielding will be required (be it additional local shielding for the magnets or beam line shutter). Implementation of a moving shutter in the beam line bears rather significant engineering and safety costs.

- Shutter in the beam line can significantly reduce dose rates to the beam line components in the direct line of sight on target.

- IR camera in the closest location to the target as assumed in the beam line design report [1] will be extremely difficult to shield down to $1 \mathrm{rad} / \mathrm{h}$. Increasing the distance and the amount of shielding material will be necessary.

- A preferred location for the OTR camera will be OTR1 as explored in this report, especially if the distance from the target and the amount of local target shielding is increased to accommodate the IR camera directly below the beam axis. One shielding enclosure for both cameras may be easier to design and reduce the amount of needed material.

\section{References:}

[1] K. Bishofberger, "Moly99 Production Facility: Report on Beamline Components, Requirements, Costs"

[2] M. Mocko, Local target shielding, LA-UR-14-29061

[3] G. Dale, private communication 